Artigo Original

Original Article

Erica Macêdo de Paula ${ }^{1}$

Debora Maria Befi-Lopes²

Descritores

Linguagem infantil

Transtornos do desenvolvimento da linguagem Comportamento social

Fonoaudiologia

Patologia da fala e linguagem

Keywords

Child language

Language developmental disorders

Social behavior

Speech, Language and Hearing Sciences Speech-language pathology

Endereço para correspondência:

Erica Macêdo de Paula

R. Cipotânea, 51, Cidade Universitária,

São Paulo (SP), Brasil, CEP: 05360-160.

E-mail: ericamdp@usp.br

Recebido em: 3/4/2012

Aceito em: 15/10/12

\section{Habilidades de resolução de conflito em crianças com Distúrbio Específico de Linguagem}

\author{
Conflict resolution abilities in children with Specific \\ Language Impairment
}

RESUMO

Objetivo: Verificar as habilidades de resolução de conflito de crianças com Distúrbio Específico de Linguagem e verificar se há correlação entre o tempo de terapia fonoaudiológica e o desempenho na tarefa de resolução de conflito. Métodos: Os sujeitos foram 20 crianças com Distúrbio Específico de Linguagem (Grupo Pesquisa) e 40 crianças em desenvolvimento normal de linguagem (Grupo Controle), com idades entre 7 anos e 8 anos e 11 meses. Para avaliar as habilidades de resolução de conflito, cinco contextos hipotéticos de conflito foram apresentados. As estratégias propostas foram assim classificadas e pontuadas em níveis: nível 0 (soluções que não se enquadram nos demais níveis); nível 1 (soluções físicas); nível 2 (soluções unilaterais); nível 3 (soluções cooperativas); e nível 4 (soluções mútuas). Resultados: A análise estatística demonstrou efeito de grupo para a variável pontuação total. Houve diferença entre os grupos para o nível de desenvolvimento modal, sendo que o Grupo Controle apresentou maior nível de desenvolvimento modal que o Grupo Pesquisa. Não houve correlação entre o tempo de terapia e a pontuação total. Conclusão: Crianças com Distúrbio Específico de Linguagem enfrentam dificuldades para resolver problemas, pois utilizam predominantemente estratégias físicas e unilaterais. Não há correlação entre o tempo de terapia e o desempenho na tarefa de resolução de conflito.

\begin{abstract}
Purpose: To investigate the conflict resolution abilities of children with Specific Language Impairment, and to verify whether the time of speech-language therapy correlates to the performance on the conflict resolution task. Methods: Participants included 20 children with Specific Language Impairment (Research Group) and 40 children with normal language development (Control Group), with ages ranging from 7 years to 8 years and 11 months. To assess the conflict resolution abilities, five hypothetical contexts of conflict were presented. The strategies used by the children were classified and scored by the following levels: level 0 (solutions that do not match the other levels), level 1 (physical solutions), level 2 (unilateral solutions), level 3 (cooperative solutions), and level 4 (mutual solutions). Results: Statistical analysis showed group effect for the variable total score. There was a difference between the groups for modal development level, with higher level of modal development observed in the Control Group. There was no correlation between the period of speech-language therapy attendance and the total score. Conclusion: Children with Specific Language Impairment present difficulties in solving problems, in view of the fact that they mainly use physical and unilateral strategies. There was no correlation between the time of speech-language therapy and performance in the task.
\end{abstract}

Trabalho realizado no Laboratório de Investigação Fonoaudiológica em Desenvolvimento da Linguagem e suas Alterações, Departamento de Fisioterapia, Fonoaudiologia e Terapia Ocupacional, Faculdade de Medicina, Universidade de São Paulo - USP - São Paulo (SP), Brasil, com bolsa concedida pela Coordenação de Aperfeiçoamento de Pessoal de Nível Superior (CAPES).

(1) Programa de Pós-graduação (Doutorado) em Ciências da Reabilitação, Departamento de Fisioterapia, Fonoaudiologia e Terapia Ocupacional, Faculdade de Medicina, Universidade de São Paulo - USP - São Paulo (SP), Brasil.

(2) Curso de Fonoaudiologia, Faculdade de Medicina, Universidade de São Paulo - USP - São Paulo (SP), Brasil. Conflito de interesse: nada a declarar. 


\section{INTRODUÇÃO}

O Distúrbio Específico de Linguagem (DEL) é diagnosticado quando há um atraso de linguagem não decorrente de nenhum outro quadro ${ }^{(1)}$. Diversos estudos relatam déficit de cognição social no DEL ${ }^{(2-4)}$.

Cognição social consiste na compreensão do mundo social, incluindo a compreensão sobre o conhecimento, pensamento, intenções, emoções e pontos de vista do outro, além do conhecimento sobre papéis e relações sociais. Um tipo de interação social que caracteriza o desenvolvimento cognitivo é a resolução de conflito. Para resolver conflitos é preciso compreender o ponto de vista do outro, assim como desenvolver e expressar estratégias para resolver disputas, portanto são necessárias habilidades cognitivas e linguísticas ${ }^{(2)}$.

A competência linguística é um importante pré-requisito para a implementação das diferentes regras sociais, porém sozinha é insuficiente. O conhecimento social básico também é necessário para a efetividade na comunicação ${ }^{(2,4)}$.

Os déficits na comunicação de crianças com DEL possivelmente são resultados de limitações gerais e não específicas da memória de trabalho e da capacidade de processamento. Essas limitações geram dificuldades de conversação e acarretam em dificuldades para compreender adequadamente interações e situações sociais, bem como a mente e o pensamento dos interlocutores. Por todas essas razões, crianças com DEL vivenciam poucas experiências sociais, o que contribui ainda mais para os déficits sociais vistos nessa população ${ }^{(5)}$. A dificuldade em realizar tarefas que exigem habilidades relacionadas às funções executivas e a dificuldade em se colocar no lugar do interlocutor provavelmente contribuem para o déficit sociocognitivo das crianças com DEL ${ }^{(6,7)}$.

As habilidades de resolução de conflito podem nos fornecer ricas e variadas informações sobre as competências linguísticas, cognitivas e sociais das crianças com DEL. Dessa forma, este estudo teve como objetivos verificar as habilidades de resolução de conflito de crianças de 7 e 8 anos de idade com DEL e verificar se há correlação entre o tempo de terapia e o desempenho na tarefa de resolução de conflito.

\section{MÉTODOS}

\section{Aspectos éticos}

Esta pesquisa foi aprovada pela Comissão de Ética para Análise de Projetos de Pesquisa do Hospital das Clínicas da Faculdade de Medicina da Universidade de São Paulo (USP), sob o n ${ }^{\circ} 0602 / 07$. Todos os responsáveis pelas crianças participantes assinaram o Termo de Consentimento Livre e Esclarecido.

\section{Casuística}

Participaram da pesquisa 60 crianças ( 37 meninas e 33 meninos) com idades variando de 7 anos a 8 anos e 11 meses. Os participantes foram divididos em dois grupos: Grupo Controle (GC) e Grupo Pesquisa (GP), na proporção de 2:1 (controle-pesquisa). O GC foi pareado ao GP em relação a gênero e idade (diferença máxima de três meses para mais ou para menos).

O GP foi constituído por 20 crianças com diagnóstico de DEL (nove meninas e 11 meninos), dez por faixa etária (7 e 8 anos). Todas as crianças do GP encontravam-se em atendimento fonoaudiológico semanal no Laboratório de Investigação Fonoaudiológica em Desenvolvimento de Linguagem e suas Alterações (LIF-DLA) do Curso de Fonoaudiologia da Faculdade de Medicina da Universidade de São Paulo na época de coleta dos dados. Os seguintes critérios de inclusão foram considerados: ter diagnóstico de DEL de acordo com critérios internacionais (alteração primária de linguagem e desenvolvimento cognitivo normal), apresentar linguagem oral e fala razoavelmente inteligível, para que as respostas pudessem ser transcritas fidedignamente.

O GC foi composto por 40 crianças sem alterações de linguagem (18 meninas e 22 meninos), 20 por faixa etária ( 7 e 8 anos). O GC foi recrutado em duas escolas públicas de ensino fundamental em áreas socioeconomicamente semelhantes da cidade de São Paulo. O critério de inclusão para o GC foi apresentar desempenho linguístico dentro da normalidade. A avaliação de linguagem para inclusão no GC incluiu os seguintes aspectos: fonologia, consciência fonológica, leitura e escrita ${ }^{(8-11)}$.

\section{Aplicação da Prova de Resolução de Conflito}

As crianças selecionadas para a pesquisa passaram por avaliação, que incluiu a Prova de Resolução de Conflito(2) (Anexo 1). Para as crianças do GC o teste foi aplicado na própria escola, em local silencioso e adequado para a aplicação, onde a avaliadora pôde aplicar a prova em cada criança individualmente. Para o GP o teste foi aplicado no LIF-DLA, antes da terapia das crianças. O teste tem duração de aproximadamente 15 minutos.

As cinco histórias que compõe a Prova de Resolução de Conflito ${ }^{(2)}$, compostas por três frases cada, foram traduzidas (translation-back translation) e apenas os nomes dos personagens foram substituídos por nomes frequentes no Português Brasileiro.

Para facilitar a compreensão das histórias foram confeccionadas 15 figuras para representar as situações hipotéticas de conflito. Para cada história foram elaboradas três figuras, cada uma representando uma frase.

Inicialmente as crianças receberam a seguinte instrução:

Eu gostaria de saber o que meninos(as) como você pensam sobre as coisas. Eu vou contar algumas coisas que aconteceram com um menino. Depois eu gostaria que você pensasse todas as coisas que ele poderia fazer. Me fale tudo o que vier na sua cabeça. Finja que todos os meninos têm [idade do sujeito] anos.

Em seguida foram apresentados oralmente às crianças cinco contextos hipotéticos de conflito descritos em artigo publicado anteriormente ${ }^{(2)}$. Ao mesmo tempo, para facilitar a compreensão das crianças, foram expostos os desenhos que representavam as situações de conflito. 
Após a apresentação de cada situação hipotética, as crianças foram instruídas a recontar a história, a fim de verificar a compreensão dos acontecimentos. Seriam excluídas do estudo as crianças que não compreendessem as situações hipotéticas de conflito, porém todos os sujeitos, de ambos os grupos, demonstraram compreender adequadamente as cinco histórias.

Logo após a recontagem de cada história, a seguinte pergunta foi feita: "Se você fosse ele [avaliadora aponta para o personagem da história], o que você faria?". Desta forma, as crianças deveriam relatar o que o protagonista poderia fazer naquela situação, fornecendo apenas uma estratégia para solução do problema.
Ao final da prova, cada criança forneceu ao todo cinco estratégias para a solução dos diferentes problemas apresentados nas cinco histórias. As respostas foram gravadas em um gravador digital Panasonic ${ }^{\circledR}$ RR-US380, para posterior análise.

As respostas foram analisadas para determinar inicialmente a presença ou ausência de estratégias de resolução de conflito. Em seguida, as respostas foram organizadas em 28 estratégias propostas no estudo em que o presente trabalho foi baseado ${ }^{(2)}$ (Quadro 1). Assim como as histórias, as estratégias de resolução foram traduzidas para o Português Brasileiro (translation - back translation).

Quadro 1. Estratégias de resolução de conflito para situações hipotéticas

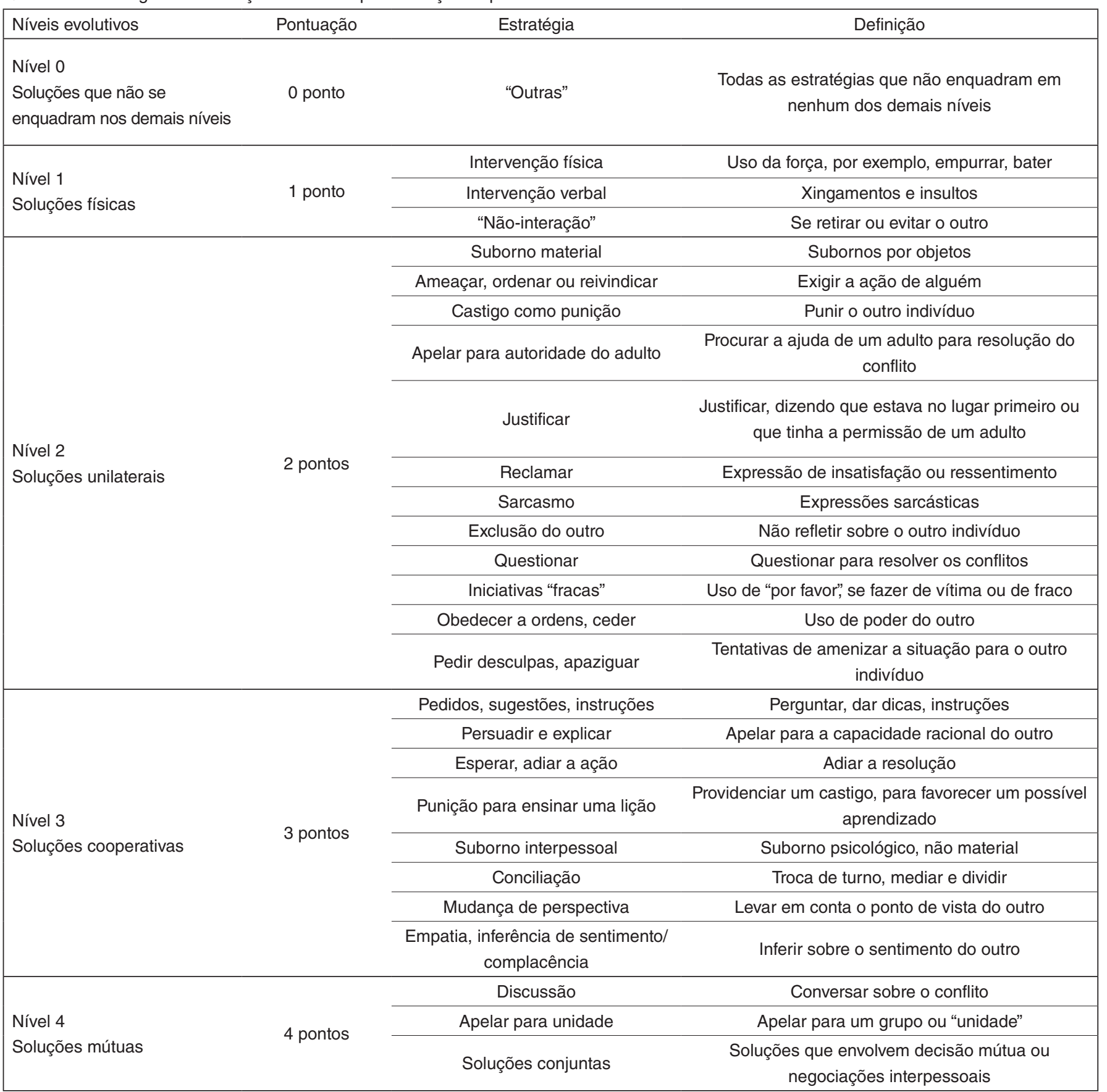


As estratégias foram agrupadas em cinco níveis que formam uma escala evolutiva de desenvolvimento para habilidades de resolução de conflitos. As respostas das crianças foram pontuadas de acordo com os níveis propostos (Quadro 1).

Para o GP foi pesquisado também o tempo de terapia de cada criança, a fim de verificar se o tempo de intervenção fonoaudiológica correlacionava-se com a pontuação na Prova de Resolução de Conflito.

\section{Análise dos dados}

Foi definido para este trabalho um nível de significância de $0,05(5 \%)$. Para realizar as análises estatísticas foram utilizados os seguintes testes: MANOVA, Mann-Whitney e Correlação de Pearson.

\section{RESULTADOS}

Para verificar os efeitos de idade e grupo na Prova de Resolução de Conflito foram atribuídos os pesos previstos para cada nível e calculada a pontuação total obtida por cada criança.

Tabela 1. Descrição da pontuação total por grupo e idade

\begin{tabular}{lcccc}
\hline Grupo & Idade & Média & Mediana & DP \\
\hline \multirow{2}{*}{ GC } & 7 anos a 7 anos e 11 meses & 10,00 & 9,50 & 2,596 \\
& 8 anos a 8 anos e 11 meses & 11,75 & 12,00 & 2,12 \\
\hline \multirow{2}{*}{ GP } & 7 anos a 7 anos e 11 meses & 6,70 & 6,50 & 2,31 \\
& 8 anos a 8 anos e 11 meses & 7,50 & 7,00 & 3,47 \\
\hline
\end{tabular}

Legenda: $\mathrm{GC}$ = grupo controle; $\mathrm{GP}=$ grupo pesquisa; $\mathrm{DP}$ = desvio-padrão

Na Tabela 1 são apresentados os resultados da estatística descritiva para a pontuação total por grupo e por idade.

Pode-se observar que a pontuação foi maior para o GC e para as crianças de 8 anos. A MANOVA foi utilizada para verificar se tais efeitos são significativos. Para a variável pontuação total, observou-se efeito de grupo $\mathrm{F}(1,52)=23,918 \mathrm{p}<0,001$, mas não de idade $F(1,52)=3,875 \mathrm{p}=0,054$. Não se observou interação entre as variáveis.

Foi determinado para cada criança o nível de desenvolvimento modal como sendo aquele em que ela usou um maior número de estratégias (Tabela 2). Para determinar diferenças no nível desenvolvimental por grupo e por idade foi utilizado o teste de Mann-Whitney. Nesta análise verificou-se diferença apenas entre os grupos $(\mathrm{p}<0,001)$, sendo que o $\mathrm{GC}$ apresentou

Tabela 2. Nível de desenvolvimento modal por grupo e idade

\begin{tabular}{llllll}
\hline & Variável & Média & Mediana & DP & Valor de $p$ \\
\hline \multirow{2}{*}{ Idade } & $\begin{array}{c}7 \text { anos a 7 anos } \\
\text { e 11 meses }\end{array}$ & 1,97 & 2,00 & 0,67 & \\
\cline { 2 - 5 } & $\begin{array}{c}8 \text { anos a 8 anos } \\
\text { e 11 meses }\end{array}$ & 2,07 & 2,00 & 0,72 & \\
\cline { 1 - 4 } Grupo & GC & 2,30 & 2,00 & 0,597 & \\
& GP & 1,45 & 1,25 & 0,48 & $<0,001^{*}$ \\
\hline
\end{tabular}

*Valores significativos $(p \leq 0,05)$ - Teste de Mann-Whitney

Legenda: $\mathrm{GC}=$ grupo controle; $\mathrm{GP}=$ grupo pesquisa; $\mathrm{DP}=$ desvio-padrão maior nível de desenvolvimento modal ( $\mathrm{M}=2,30 \pm 0,597)$ do que o GP $(\mathrm{M}=1,45 \pm 0,48)$.

Foi examinada também a proporção de estratégias usadas em cada um dos cinco níveis propostos, tendo sido calculada para cada criança dividindo o número de estratégias de cada nível pelo número total de estratégias utilizadas pela criança. Para determinar as diferenças por grupo e por idade e entre os grupos, foi utilizado o teste de Mann-Whitney. No GC as crianças de 7 anos utilizaram mais os níveis 1 $(\mathrm{M}=0,21 \pm 0,2), 2(\mathrm{M}=0,52 \pm 0,25)$ e $3(\mathrm{M}=0,21 \pm 0,24)$, e as de 8 anos os níveis $2(\mathrm{M}=0,52 \pm 0,25)$ e $3(\mathrm{M}=0,4 \pm 0,23)$ (Tabela 3). Houve diferença para os níveis 1 (mais utilizado

Tabela 3. Proporção de estratégias utilizadas de acordo com os cinco níveis propostos por faixa etária para o grupo controle

\begin{tabular}{lccc}
\hline Nível & & 7 anos & 8 anos \\
\hline \multirow{2}{*}{ Nível 0 } & Média & 0,03 & 0,04 \\
& Mediana & 0,00 & 0,00 \\
Nível 1 & DP & 0,07 & 0,09 \\
& Média & 0,21 & 0,04 \\
& Mediana & 0,20 & 0,00 \\
Nível 2 & DP & 0,2 & 0,11 \\
& Média & 0,52 & 0,52 \\
& Mediana & 0,60 & 0,40 \\
Nível 3 & DP & 0,25 & 0,25 \\
& Média & 0,21 & 0,4 \\
& Mediana & 0,20 & 0,40 \\
Nível 4 & DP & 0,24 & 0,23 \\
& Média & 0,03 & 0,03 \\
\hline
\end{tabular}

Legenda: $\mathrm{DP}=$ desvio-padrão

pelas crianças de 7 anos; $p=0,001$ ) e 3 (mais utilizado pelas crianças de 8 anos; $p=0,015$ ).

É possível observar que, no GP, tanto as crianças de 7 anos quanto as de 8 anos utilizaram com mais frequência os níveis 1 ( 7 anos: $\mathrm{M}=0,31 \pm 0,36 ; 8$ anos: $\mathrm{M}=0,51 \pm 0,27$ ) e 2 ( 7 anos: $M=0,45 \pm 0,34 ; 8$ anos: $M=0,35 \pm 0,23$ ). Para este grupo, observou-se diferença apenas no nível $0(p=0,020)$, em que as crianças de 7 anos usaram mais estratégias que as de 8 anos (Tabela 4).

$\mathrm{Na}$ Tabela 5 são apresentados os resultados da comparação entre os grupos por idade e nível, quanto à proporção de estratégias utilizadas. Verificou-se diferença no nível 0 para a faixa etária de 7 anos, em que predomina o uso de estratégias neste nível para o GP. Para a faixa etária de 8 anos, observou-se diferença para os níveis 1 e 3, sendo que para o GC predominaram estratégias no nível 3 e para o GP no nível 1.

Foi realizada a Correlação de Pearson para verificar se havia correlação entre o tempo de terapia fonoaudiológica e o desempenho das crianças na Prova de Resolução de 
Tabela 4. Proporção de estratégias utilizadas de acordo com os cinco níveis propostos por faixa etária para o grupo pesquisa

\begin{tabular}{lccc}
\hline Nível & & 7 anos & 8 anos \\
\hline \multirow{3}{*}{ Nível 0 } & Média & 0,18 & 0,05 \\
& Mediana & 0,20 & 0,00 \\
Nível 1 & DP & 0,10 & 0,09 \\
& Média & 0,31 & 0,51 \\
Nível 2 & DP & 0,30 & 0,47 \\
& Média & 0,45 & 0,27 \\
\hline \multirow{3}{*}{ Nível 3 } & Mediana & 0,50 & 0,35 \\
& DP & 0,34 & 0,23 \\
\hline \multirow{3}{*}{ Nível 4 } & Média & 0,05 & 0,08 \\
& Mediana & 0,00 & 0,00 \\
& DP & 0,09 & 0,14 \\
\hline
\end{tabular}

Legenda: DP = desvio-padrão

Tabela 5. Estatística inferencial para a comparação da proporção de estratégias utilizadas pelos grupos controle e pesquisa de acordo com os cinco níveis propostos por faixa etária

\begin{tabular}{lccccc}
\hline Faixa etária & Nível 0 & Nível 1 & Nível 2 & Nível 3 & Nível 4 \\
\hline 7 anos & $<0,001^{*}$ & 0,712 & 0,804 & 0,053 & 1,00 \\
8 anos & 0,838 & $<0,001^{*}$ & 0,081 & $0,001^{*}$ & 0,655 \\
\hline
\end{tabular}

*Valores significativos $(p \leq 0,05)$ - Teste de Mann-Whitney

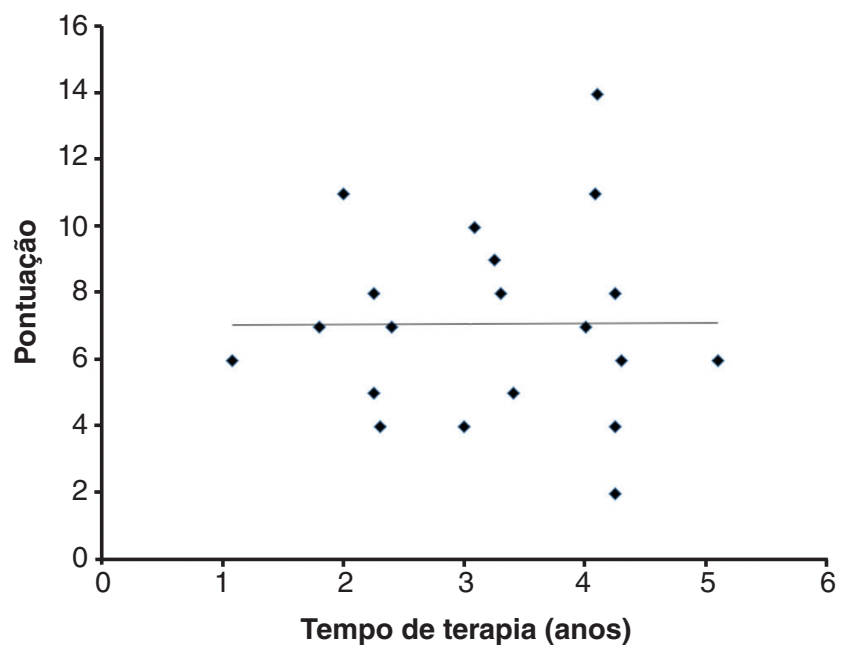

Figura 1. Correlação entre o tempo de terapia e a pontuação total na Prova de Resolução de Conflito

Conflito. Não houve correlação entre o tempo de terapia e a pontuação total na prova $(\mathrm{p}=0,961 ; \mathrm{r}=0,012)$ (Figura 1$)$.

\section{DISCUSSÃO}

Era esperado que as crianças com distúrbio de linguagem apresentassem pior desempenho quando comparadas às crianças em desenvolvimento normal de linguagem na Prova de Resolução de Conflito, devido ao atraso linguístico, às dificuldades de processamento, ao déficit sociocognitivo e à dificuldade de se colocar no lugar do interlocutor, que são característicos do $\mathrm{DEL}^{(7,12-16)}$.

De fato, as crianças com DEL participantes da pesquisa obtiveram na Prova de Resolução de Conflito uma pontuação total significativamente inferior à de crianças em desenvolvimento normal de linguagem. Portanto, os achados deste estudo comprovam que crianças com DEL apresentam atraso no desenvolvimento de habilidades sociocognitivas e têm dificuldade para solucionar problemas de maneira adequada, resultado esse que corrobora diversas pesquisas realizadas com essa população ${ }^{(2,4,17,18)}$.

$\mathrm{O}$ fato de crianças com DEL apresentarem importantes dificuldades com atividades sociais corriqueiras, como a solução de problemas, contribui para a impopularidade de grande parte dessas crianças com os pares da mesma idade, no que diz respeito às amizades, relações e interações ${ }^{(19,20)}$. Além disso, as dificuldades sociais e escolares que as crianças com DEL enfrentam ao longo da vida contribuem para a baixa autoestima dessa população, característica essa que foi observada em diversos trabalhos ${ }^{(20-22)}$.

Apesar do déficit sociocognitivo no DEL ser evidente, ainda não é possível estabelecer a exata relação entre linguagem e cognição social. O que se sabe é que as dificuldades de comunicação das crianças com atraso linguístico interferem nas relações sociais e, consequentemente, agravam os déficits sociocognitivos, uma vez que as crianças passam a interagir menos que os seus pares em desenvolvimento normal e possuem menos chances de aplicar as regras sociais ${ }^{(5,23)}$.

Pesquisas futuras poderiam também investigar as relações entre teoria da mente, funções executivas e resolução de conflito no DEL, pois todas essas habilidades parecem estar relacionadas. Se de fato essa relação for confirmada, pode influenciar as intervenções terapêuticas de maneira significativa ${ }^{(6,7)}$.

Tanto no desenvolvimento normal de linguagem quanto no DEL não houve diferença entre a pontuação total das crianças de 7 anos o que indica que as crianças dessa faixa etária resolvem conflitos da mesma maneira. Já era esperado que o desempenho entre as faixas etárias se mantivesse constante, uma vez que as habilidades linguísticas e sociocognitivas não evoluem significativamente entre essas faixas etárias.

Apesar da pontuação não ter diferido entre as faixas etárias, as crianças em desenvolvimento normal de 7 anos utilizaram com mais frequência estratégias do Nível 1, e as de 8 anos utilizaram mais vezes estratégias do Nível 3. Provavelmente as crianças de 8 anos conseguiram utilizar consistentemente as estratégias cooperativas pois, além da maior experiência com situações sociais, somente por volta dessa idade as crianças passam a compreender de maneira efetiva os pensamentos como sendo interpretações que podem variar entre indivíduos e de acordo com as situações. Além disso, também é nessa época que as crianças adquirem a capacidade de raciocinar sobre múltiplas possibilidades e de responder a perguntas do tipo "e se"; habilidades importantes para a solução de problemas ${ }^{(24,25)}$. 
Um estudo realizado em 2008 mostrou evidências de que a interação entre teoria da mente e funções executivas continua se desenvolvendo até o final da adolescência. Como há uma importante relação entre essas habilidades e a capacidade de solucionar problemas, acredita-se que as habilidades de solução de problemas evoluam consideravelmente após os 8 anos $^{(26)}$.

Entre as crianças com DEL, a única diferença observada entre os níveis de respostas foi para o Nível 0, mais utilizado pelas crianças de 7 anos. Não é possível afirmar com certeza o motivo dessa diferença, mas ela provavelmente ocorreu pois as crianças de 8 anos possuem mais experiência com situações sociais do que as crianças de 7 anos.

De acordo com o exposto, é provável que a evolução das habilidades de cognição social e, consequentemente, a evolução da capacidade para solucionar problemas, esteja relacionada às experiências sociais individuais em conjunto com a evolução da linguagem e da capacidade de raciocínio abstrato, tanto para as crianças com DEL quanto para as crianças em desenvolvimento normal.

Na Prova de Resolução de Conflito as crianças com DEL utilizaram com mais frequência estratégias unilaterais (Nível 2) e soluções físicas (Nível 1), enquanto as crianças em desenvolvimento normal de linguagem utilizaram, além das estratégias dos Níveis 1 e 2, estratégias cooperativas (Nível 3). Dessa forma, além da diferença observada na pontuação total, as crianças com DEL também apresentaram menor nível de desenvolvimento modal $(\mathrm{GP}=1,45 ; \mathrm{GC}=2,30)$.

Esse dado demonstra que apesar dos dois grupos terem utilizado estratégias unilaterais (Nível 2) para solucionar os problemas apresentados, as crianças dessa faixa etária também utilizam com grande frequência estratégias que envolvem intervenção física (Nível 1). Porém, as crianças em desenvolvimento normal de 8 anos já são capazes de utilizar com consistência estratégias cooperativas (Nível 3).

Segundo os resultados encontrados, crianças de 8 anos com DEL ainda utilizam com frequência estratégias agressivas (físicas ou verbais) para solucionar problemas. Ao contrário dos resultados encontrados nesta pesquisa, a maioria dos estudos que avaliou o comportamento de crianças com DEL não observou comportamentos agressivos, e sim comportamentos de retirada e de esquiva ${ }^{(23,27)}$. É provável que as crianças com DEL, por não conseguirem utilizar estratégias de resolução de conflito mais sofisticadas e efetivas, acabam utilizando estratégias como xingar e bater. Possivelmente, com o passar dos anos, elas percebem que esse tipo de comportamento não é efetivo e acabam optando por estratégias como se retirar ou evitar o outro.

Não foi observada correlação significativa entre o desempenho na tarefa de resolução de conflito e o tempo de terapia fonoaudiológica, provavelmente porque o desenvolvimento das habilidades sociocognitivas envolve aspectos linguísticos e sociais e, apesar de o foco da terapia fonoaudiológica recair sobre os aspectos linguísticos, ela consiste num contexto artificial e isoladamente não é capaz de suprir os déficits sociais frequentemente observados na população com DEL.

Os resultados desta pesquisa sugerem que os deficits sociocognitivos observados na população com DEL possivelmente não decorrem exclusivamente do distúrbio de linguagem. Desta forma, crianças com DEL se beneficiariam de estratégias de intervenção terapêutica que visassem o uso da linguagem em diferentes contextos sociais para, desta forma, aumentar as oportunidades das crianças de aprimorar as habilidades sociais de comunicação em situações reais. Esse tipo de intervenção favoreceria as interações dessas crianças, uma vez que o sucesso em tarefas sociais e na resolução de conflito é essencial para o estabelecimento e a manutenção de relações interpessoais positivas.

Por ser provável que crianças mais velhas e adolescentes utilizem com mais frequência estratégias mais sofisticadas, seria interessante aumentar a amostra da pesquisa, acrescentando mais faixas etárias. Desta forma será possível traçar um perfil detalhado para a evolução das habilidades de resolução de conflito.

\section{CONCLUSÃO}

Este estudo demonstrou que crianças com DEL enfrentam dificuldades para resolver problemas, pois utilizam predominantemente estratégias físicas e unilaterais que não são eficientes para solucionar a grande maioria dos conflitos vivenciados por crianças de 7 e 8 anos de idade.

Quanto às comparações entre as faixas etárias estudadas, verificou-se que as crianças de 7 e 8 anos em geral (seja em desenvolvimento normal de linguagem ou com DEL) utilizam estratégias semelhantes para solucionar conflitos. Da mesma forma, não foi observada diferença quantitativa entre os gêneros para a tarefa de resolução de conflito.

O distúrbio de linguagem foi a única variável que determinou o desempenho na Prova de Resolução de Conflitos, pois o GP obteve desempenho inferior ao GC. Além disso, não foi observada correlação entre o desempenho na prova e o tempo de terapia fonoaudiológica, sugerindo que a terapia de linguagem isoladamente não é suficiente para adequar o déficit sociocognitivo observado na população com DEL.

\footnotetext{
* EMP foi responsável pela coleta, tabulação dos dados e elaboração do manuscrito; DMBL supervisionou a coleta de dados, foi responsável pelo projeto, delineamento do estudo e orientação geral das etapas de execução e elaboração do manuscrito.
}

\section{REFERÊNCIAS}

1. Befi-Lopes DM. Avaliação diagnóstica e aspectos terapêuticos nos Distúrbios Específicos de Linguagem. In: Fernandes FDM, Mendes BCA, Navas ALGP, organizadores. Tratado de Fonoaudiologia. São Paulo: Roca; 2010. p. 314-22.

2 Stevens LJ, Bilss LS. Conflict resolution abilities of children with Specific Language Impairment and children with normal language. J Speech Hear Res. 1995;38:599-611.

3. Brinton B, Spackman MP, Fujiki M, Ricks J. What Should Chris Say? The Ability of Children With Specific Language Impairment to Recognize the Need to Dissemble Emotions in Social Situations. J Speech Lang Her Res. 2007;50:798-811.

4. Merkenschlager A, Amorosa H, Kiefl H, Martinius J. Recognition of Face Identity and Emotion in Expressive Specific Language Impairment. Folia Phoniatr Logop. 2012;24;64:73-9. 
5. Redmond SM, Rice ML. The socioemotional behaviors of children with SLI: Social Adaptation or Social Deviance? J Speech Lang Hear Res. 1998;41:688-700.

6. Thirion-Marissiaux AF, Nader-Grosbois N. Theory of mind "beliefs", developmental characteristics and social understanding in children and adolescents with intellectual disabilities. Res Dev Disabil. 2008;29:41430.

7. Hughes DM, Turkstra L, Wulfeck BB. Parent and self-ratings of executive function in adolescents with specific language impairment. Int J Lang Commun Disord. 2009;19:901-16.

8. Wertzner HF. Fonologia. In: Andrade CRF, Befi-Lopes DM, Fernandes FDM, Wertzner HF. Teste de Linguagem Infantil ABFW. Carapicuíba: Pro Fono. 2004. p. 5-31.

9. Rosal CAR. Habilidades de segmentação fonêmica em crianças normais de primeira, segunda e terceira séries do ensino fundamental [dissertação]. São Paulo: Faculdade de Medicina da Universidade de São Paulo; 2002.

10. Herrero SF. Perfil das crianças: pré-escolares e escolares no teste de sensibilidade fonológica [dissertação]. São Paulo: Faculdade de Medicina da Universidade de São Paulo; 2001.

11 Andrade CRF, Befi-Lopes DM, Fernandes, FDM, Wertzner HF. Manual de avaliação de Linguagem do serviço de Fonoaudiologia do centro de saúde escola Samuel B. Pessoa [publicação interna]. São Paulo: Centro de Saúde Escola Samuel B. Pessoa; 1997.

12. Befi-Lopes DM, Rodrigues A, Rocha LC. Habilidades linguístico-pragmáticas em crianças normais e com alteração no desenvolvimento da linguagem. Pro Fono. 2004;16:57-66.

13. Befi-Lopes DM, Rocha LC. Análise pragmática das respostas de crianças com e sem Distúrbio Específico de Linguagem. Pro Fono. 2006;18:229-38.

14. Rocha LC, Befi-Lopes DM. Análise de iniciativa comunicativa em crianças com desenvolvimento normal de linguagem e com Distúrbio Específico de Linguagem (DEL). Rev Soc Bras Fonoaudiol. 2006; 11:28-36

15. Buiza-Navarrete JJ, Adrian-Torres JA, Gonzalez-Sanchez M. Neurocognitive markers in specific language impairment. Rev Neurol 2007;44:326-33.
16. Farrar MJ, Johnson B, Tompkins V, Easters M, Zilisi-Medus A, Benigno JP. Language and theory of mind in preschool children with specific language impairment. J Commun Disord. 2009;42:428-41.

17. Hart KI, Fujiki M, Brinton B, Hart CH. The relationship between social behavior and severity of language impairment. J Speech Lang Hear Res. 2004;47:647-62.

18. Marton K, Abramoff B, Rosenzweig S. Social cognition and language in children with specific language impairment. J Commun Disord. 2005;38:143-62.

19. Durkin K, Conti-Ramsden G. Language, social behavior, and the quality of friendships in adolescents with and without a history of specific language impairment. Child Dev. 2007;78:1441-57.

20. Redmond SM. Peer victimization among students with specific language impairment, attention-deficit/hyperactivity disorder, and typical development. Lang Speech Hear Serv Sch. 2011;42:520-35.

21. Fujiki M, Spackman MP, Brinton B, Hall A. The relationship of language and emotion regulation skills to reticence in children with specific language impairment. J Speech Lang Hear Res. 2004;47:637-46.

22. Wadman R, Durkin K, Conti-Ramsden G. Self-esteem, shyness, and sociability in adolescents with specific language impairment (SLI). J Speech Lang Hear Res. 2008;51:938-52.

23. Horowitz L, Jansson L, Ljungberg T, Hedenbro M. Behavioural patterns of conflict resolution strategies in preschool boys with language impairment in comparison with boys with typical language development. Int J Lang Commun Disord. 2005;40:431-54.

24. Apperly IA, Robinson EJ. When can children handle referential opacity? Evidence for systematic variation in 5- and 6-year-old children's reasoning about beliefs and belief reports. J Exp Child Psychol. 2003;85:297311.

25. Beck SR, Robinson EJ, Carroll DJ, Apperly IA. Children's thinking about counterfactuals and future hypotheticals as possibilities. Child Dev. 2006;77:413-26.

26. Dumontheil I, Apperly IA, Blakemore SJ. Online usage of theory of mind continues to develop in late adolescence. Dev Sci. 2010;13:331-8.

27. Horowitz L, Westlund K, Ljungberg T. Aggression and withdrawal related behavior within conflict management progression in preschool boys with language impairment. Child Psychiatry Hum Dev. 2007;38:237-53. 
Anexo 1. Situações hipotéticas de conflito

1. Matheus é o melhor amigo de João. Mas agora Matheus brinca com um novo menino da escola todos os dias. Matheus nunca mais brinca com João.

2. Rodrigo quer usar o computador para jogar seu jogo favorito. Seu irmão Lucas já está usando o computador. Lucas odeia ser interrompido quando está usando o computador.

3. Marcelo está com fome. Ele quer que seu irmão mais velho, Daniel, o ajude a pegar alguns biscoitos que estão em uma prateleira alta. Marcelo está com medo que Daniel diga não.

4. Tem um menino chamado Marcos que mora perto do Eduardo. Marcos é muito chato. Quase todos os dias Marcos xinga Eduardo no caminho para escola.

5. Pedro é um novo menino no bairro. Um sábado Bruno chama Pedro para assistir desenho animado. Depois de dez minutos Pedro muda de canal sem perguntar. 